\title{
Ultrasound-Guided Emergency Endoscopic Retrograde Biliary Drainage Without Radiography
}

In most hospitals, endoscopic retrograde cholangiopancreatography (ERCP) is carried out in the endoscopic or radiological unit. However, transporting critically ill patients and their monitoring equipment outside of the intensive care unit can create considerable logistical problems. Many intensive care units are not equipped with quality fluoroscopy facilities for conducting ERCP examinations, although an ultrasound machine should be easily made available in most.

We recently treated two patients with biliary obstruction who were thought to be too ill to undergo ERCP in our endoscopic unit. The first patient, a 67-year-old woman with suspected obstructive cholangitis, was hemodynamically unstable and under artificial ventilation due to respiratory failure. The second patient was a 76year-old woman who presented with severe abdominal pain and spiking fever several hours after an endoscopic sphincterotomy for papillary stenosis. Since the pain could only be controlled by intravenous morphine, she was transferred to the intensive care unit.

With the patients lying on their left side, the duodenoscope was advanced to the papilla. The orifice was gently cannulated using a standard ERCP catheter. A guide wire was then advanced through the cannula into the common bile duct (Figure 1). After clea visualization of the wire in the common duct, a pigtail cathete was placed in the first patient, and a straight catheter in the second Ultrasound examination showed marked aerobilia, confirming the correct positioning of the biliary prostheses (Figure 2). Since hemobilia was the cause of obstruction in the second patient, ar additional nasobiliary tube was inserted to allow flushing of thi biliary system. In both patients, satisfactory biliary drainage wa: achieved within 20 minutes of starting the procedure. Both patient: tolerated the examination well.

The technique appears to be very suitable for severely ill patient: who cannot be moved from the intensive care unit. We believe i should be attempted before more risky percutaneous techniques such as ultrasound-guided percutaneous transhepatic biliary 0 gallbladder drainage, are used, particularly in patients witl coagulation disorders $(1,2)$. In addition, ultrasound-guided retro grade biliary drainage can also be used in early pregnancy, and it cases of thyrotoxicosis or severe iodine allergy when roentgen ra: or iodine exposure should be avoided.

T. F. Schorn, U. Jahns, C. Vollmer, R. Gugler Medical Clinic I, City Clinic, Karlsruhe, Germany 


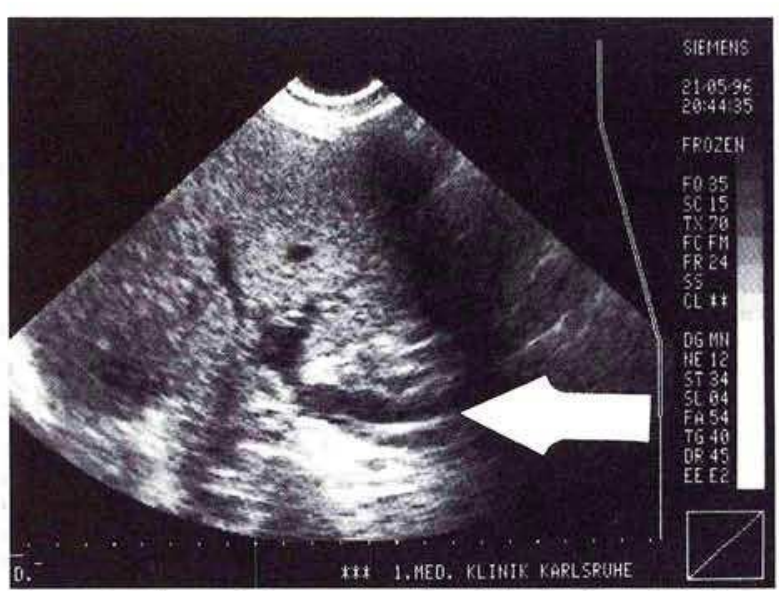

Figure 1: Ultrasound demonstrates the guide wire (arrow) inside the dilated common bile duct.

\section{References}

1. Das K, Kochhar R, Mehta SK, Suri S. A modified technique of ultrasonically guided percutaneous transhepatic biliary drainage. Surg Endosc 1989; 3: 191-4.

2. Maurya SD, Agarwal MS, Bhadauria RP, et al. Ultrasoundguided percutaneous cholecystostomy. Int Surg 1991; 76: 189-91.

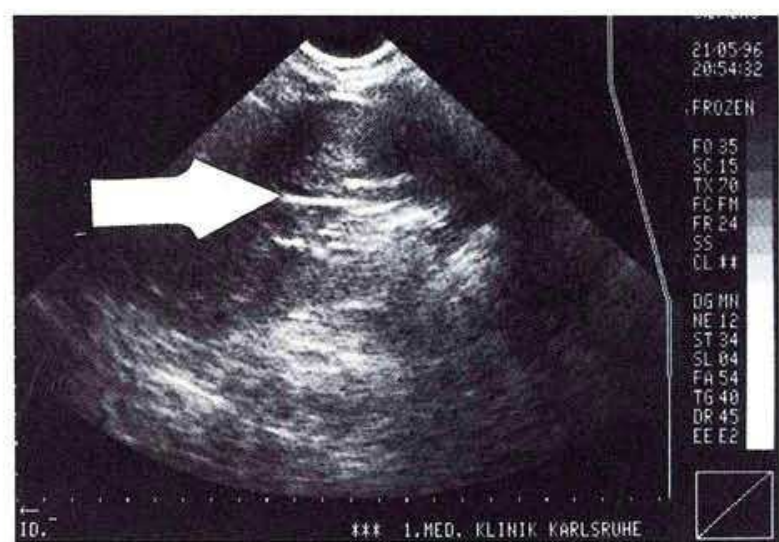

Figure 2: Aerobilia (arrow) confirms adequate biliary drainage.

\section{Corresponding Author}

T. F. Schorn, M.D., I. Medizinische Klinik

Städtisches Klinikum Karlsruhe

Akademisches Lehrkrankenhaus

der Universität Freiburg, Postfach 6280

76042 Karlsruhe, Germany

Fax: +49-721-974-2709 